\title{
GORENSTEIN ALGEBRAS AND THE CAYLEY-BACHARACH THEOREM
}

\author{
E. D. DAVIS, A. V. GERAMITA AND F. ORECCHIA
}

\begin{abstract}
This paper is an examination of the connection between the classical Cayley-Bacharach theorem for complete intersections in $\mathbf{P}^{2}$ and properties of graded Gorenstein algebras.
\end{abstract}

Introduction. It is known, if not well known, that the Cayley-Bacharach theorem for complete intersections in $\mathbf{P}^{2}$ is valid for 0 -dimensional arithmetically Gorenstein subschemes of $\mathbf{P}^{n}$. More generally, we show that the result is valid for 0 -dimensional subschemes of $\mathbf{P}^{n}$ having minimal Cohen-Macaulay type compatible with their Hilbert functions. The Cayley-Bacharach theorem in the Gorenstein case is a special instance of a theorem, interesting and technically useful in its own right, relating the Hilbert functions of linked subschemes of $\mathbf{P}^{n}$. Lastly we show that the 0 -dimensional, arithmetically Gorenstein, reduced subschemes of $\mathbf{P}^{n}$ are characterized by the validity of the Cayley-Bacharach theorem and the symmetry of the Hilbert function.

Fixed notation. $A$ denotes a standard $\mathbf{N}$-graded $k$-algebra, $k$ a field: $A_{0}=k$; $A=k\left[A_{1}\right] ; \lambda\left(A_{1}\right)<\infty$. We use $\lambda$ to denote $k$-linear dimension, reserving "dim" for dimension of rings or schemes, and $\delta$ to denote multiplicity for such algebras (or degree of the corresponding projective scheme). Note that $\delta(A)=\lambda(A)$ if $\operatorname{dim} A=0$. We use $I$ to denote a nonzero, nonunit, homogeneous ideal of $A$, and $J=$ ann $I$ ("ann" = annihilator). We assume always that $A$ and $A / I$ are CM (Cohen-Macaulay) and that $\operatorname{Ass}(A / I) \subset \operatorname{Ass}(A)$. Hence Ass $(A / J) \subset \operatorname{Ass}(A)$. (Indeed, since the 0-ideal of $A$ is unmixed of height 0 , so is the annihilator of any nonzero ideal of $A$.) Therefore $A / J$ is CM if $\operatorname{dim} A \leqslant 1$. In any case, $A / J$ is CM if $A$ is Gorenstein [PS, Proposition 1.3]. In our applications $A / J$ will be $\mathrm{CM}$ for one of these two reasons.

Recall that, by definition, a ring $R$ is Gorenstein provided that $R_{p h}$ is a Gorenstein local ring for every prime ideal $\not h$ of $R$, and $A$ is Gorenstein $\Leftrightarrow A_{A_{1} A}$ is a Gorenstein local ring $[\mathbf{A G}]$. We refer to $[\mathbf{K}]$ for those properties of Gorenstein local rings which are used below without specific reference.

1. Observations. Assume that $A_{p}$ is Gorenstein for all $\left.p \in \operatorname{Ass}(A)\right)$. Then:

(a) $\delta(A)=\delta(A / I)+\delta(A / J)$.

Received by the editors January 3, 1984.

1980 Mathematics Subject Classification. Primary 13H10, 14 N10.

Kev words and phrases. Arithmetically Cohen-Macaulay subschemes of $\mathbf{P}^{n}$, Gorenstein, Cohen-Macaulay type, Cayley-Bacharach, Hilbert function, algebraic liaison, conductor.

$(1985$ American Mathematical Society $0002-9939 / 85 \$ 1.00+\$ .25$ per page 
(b) Suppose $\operatorname{dim} A=0$, and let $N=\max \left\{t \in \mathbf{N} \mid A_{t} \neq 0\right\}$. Then

$$
\lambda\left(A_{t}\right)=\lambda\left(I_{t}\right)+\lambda\left(J_{N-t}\right)=\lambda\left(A_{N-t}\right), \quad 0 \leqslant t \leqslant N .
$$

Proof. (a) Since $A_{\not}$ is a 0 -dimensional Gorenstein local ring for $\not p \in \operatorname{Ass}(A)$,

$$
\text { length }\left(A_{p}\right)=\text { length }\left(A_{p} / I A_{p}\right)+\operatorname{length}\left(A_{p} / J A_{p}\right) \text {. }
$$

Now use the multiplicity formula [N, p. 76]:

$$
\begin{aligned}
\delta(A) & =\sum \delta(A / \not) \text { length }\left(A_{\not h}\right) \quad(\text { sum over } \not p \in \operatorname{Ass}(A)) \\
& =\sum \delta(A / \not) \text { length }\left(A_{p} / I A_{\not}\right)+\sum \delta(A / \not) \operatorname{length}\left(A_{\not} / J A_{\not}\right) \\
& =\delta(A / I)+\delta(A / J) .
\end{aligned}
$$

(b) Since $A$ is a 0 -dimensional Gorenstein local $\operatorname{ring}, \lambda\left(\operatorname{ann}\left(A_{1}\right)\right)=1$. Hence ann $\left(A_{1}\right)=A_{N}$. It follows easily that the $k$-bilinear map $A_{i} \times A_{j} \rightarrow A_{i+j}$, induced by multiplication, is nonsingular for $i+j \leqslant N$. From this one deduces that $\left(\operatorname{ann}\left(I_{t}\right)\right)_{N-t}=J_{N-t}$. (b) now follows easily from the nonsingular $k$-bilinear pairing $A_{t} \times A_{N-t} \rightarrow A_{N} \cong k$.

Observation 1(b), a well-known property of quasi-Frobenius algebras, contains our theorem relating the Hilbert functions of linked projective schemes. To see this we require certain standard technicalities.

Further notation. $H(S,-)$ denotes the Hilbert function of the standard $\mathbf{N}$-graded $k$-algebra $S$ (i.e., $H(S, t)=\lambda\left(S_{t}\right)$ ), and $\Delta$ denotes the difference operator on $\mathbf{Z}$-valued sequences (i.e., if $f$ is a $\mathbf{Z}$-valued sequence, then $\Delta f(0)=f(0)$ and $\Delta f(i)=f(i)-$ $f(i-1)$ for $i>0)$. Since $H(S, t)$ is a degree $\operatorname{dim} S-1$ polynomial function of $t$ for $t \gg 0, \Delta^{\operatorname{dim} S} H(S, t)=0$ for $t \gg 0$. Define:

$$
\sigma(S)=1+\max \left\{t \in \mathbf{N} \mid \Delta^{\operatorname{dim} S} H(S, t) \neq 0\right\} .
$$

Observe that for any $t \geqslant \sigma(S)-1, \delta(S)=\sum\left\{\Delta^{\operatorname{dim} S} H(S, j) \mid 0 \leqslant j \leqslant t\right\}$, and $\delta(S)=$ $\Delta^{\operatorname{dim} S-1} H(S, t)$ if $\operatorname{dim} S>0$. For any nonzero, nonunit, homogeneous ideal $Q$ of $S$, define:

$$
\sigma(Q)=\sigma(S / Q) ; \quad \alpha(Q)=\min \left\{t \in \mathbf{N} \mid Q_{t} \neq 0\right\} .
$$

Observe that for any $m \in \mathbf{N}, \alpha(Q)=\min \left\{t \in \mathbf{N} \mid \Delta^{m} H(S / Q, t) \neq \Delta^{m} H(S, t)\right\}$.

Reduction to dimension 0 . Henceforth, for technical convenience, we assume $k$ to be infinite, in which case there is an $A_{1} A$-primary ideal $Q$ generated by an $A$-regular sequence in $A_{1}$. (So this sequence is also $(A / I)$ )-regular and, if $A / J$ is $\mathrm{CM}$, then $(A / J)$-regular.) Let $x \mapsto \bar{x}$ denote the canonical map $A \rightarrow A / Q=\bar{A}$. By [G], $\lambda\left(\operatorname{ann}\left(\overline{A_{1}}\right)\right)$ is independent of the choice of $Q$; this integer is called the CM-type of $A$. Recall that $A$ is Gorenstein $\Leftrightarrow \bar{A}$ is Gorenstein $\Leftrightarrow$ CM-type of $A=1$.

2. ObServations. Let $m=\operatorname{dim} A$.

(a) $\Delta^{m} H(A,-)=H(\bar{A},-) ; \Delta^{m} H(A / I,-)=H(\bar{A} / \bar{I},-) ; \bar{I} \neq(0)$.

(b) $\delta(A)=\delta(\bar{A})=\lambda(\bar{A}) ; \delta(A / I)=\delta(\bar{A} / \bar{I})=\lambda(\bar{A} / \bar{I})$.

(c) $\sigma(A)=\sigma(\bar{A}) ; \sigma(I)=\sigma(\bar{I}) ; \alpha(I)=\alpha(\bar{I})$.

(d) $\alpha(\bar{I}) \leqslant \sigma(\bar{I}) \leqslant \sigma(\bar{A}) \neq \alpha(\bar{I})$. 
Proof. (a) follows immediately from the fact that $Q$ is generated by a sequence which is both $A$ - and ( $A / I)$-regular, and (b)-(d) follow formally from (a) and definitions.

3. Theorem (Hilbert Functions Under Liaison). Suppose $A$ is Gorenstein. Let $m=\operatorname{dim} A, N=\sigma(A)-1$. Then:

(a) $\Delta^{m} H(A, t)=\Delta^{m} H(A, N-t), 0 \leqslant t \leqslant N$.

(b) $\Delta^{m} H(A, t)=\Delta^{m} H(A / I, t)+\Delta^{m} H(A / J, N-t), 0 \leqslant t \leqslant N$.

(c) $\alpha(I)+\sigma(J)=\alpha(J)+\sigma(I)=\sigma(A)$.

Proof. First note that (c) is a formal consequence of (b) and definitions, and that (a) and (b) follow immediately from 1(b) if $m=0$. Hence (a) and (b) follow immediately from $2(\mathrm{a}, \mathrm{c})$, the 0 -dimensional case, and

Claim. $\bar{J}=$ ann $\bar{I}$.

Proof. By 2(b) and 1(a)

$$
\begin{aligned}
\lambda(\bar{A} / \bar{J}) & =\delta(A / J)=\delta(A)-\delta(A / I)=\delta(\bar{A})-\delta(\bar{A} / \bar{I}) \\
& =\delta(\bar{A} / \operatorname{ann} \bar{I})=\lambda(\bar{A} / \operatorname{ann} \bar{I}) .
\end{aligned}
$$

Since $\bar{J} \subseteq$ ann $\bar{I}, \bar{J}=$ ann $\bar{I}$, and we are done.

RemarK. Suppose $A$ is Gorenstein. Then, as a corollary to 3(c), we have the validity of the Cayley-Bacharach theorem for A:

$$
\delta(A / I)=\delta(A)-1 \Rightarrow \alpha(I)=\sigma(A)-1 .
$$

(Proof. $\delta(A / J)=1$, whence $\sigma(J)=1$.) We call this result "Cayley-Bacharach" because: specializing to the case in which $\operatorname{Proj}(A)$ is the complete intersection of two curves in $\mathbf{P}^{2}$, in which case $\sigma(A)$ is one less than the sum of the degrees of the curves, we obtain the classical Cayley-Bacharach theorem. (See [SR, pp. 97-101] for further details.) More generally, 3(c) gives: $\operatorname{Proj}(A / I)$ is in generic position in $\operatorname{Proj}(A) \Leftrightarrow \operatorname{Proj}(A / J)$ is in generic position in $\operatorname{Proj}(A)$. ("Generic position" simply means " $\sigma \leqslant \alpha+1$ "; see [O] for a geometric interpretation of "generic position in $\mathbf{P}^{n}$ ".) The validity of Cayley-Bacharach for $A$ is, in fact, a consequence of only "half" of the Gorenstein property, since more generally we have

4. TheOREM. $\Delta^{\operatorname{dim} A} H(A, \sigma(A)-1) \leqslant C M$-type of $A$. If equality holds, then

$$
\delta(A / I) \leqslant \delta(A)-\sigma(A)+\alpha(I) \leqslant \delta(A)-1 .
$$

Proof (CF. [DM, PROOF OF (2.3)]). CM-type of $A=\lambda\left(\operatorname{ann} \overline{A_{1}}\right) \geqslant \lambda\left(\overline{A_{N}}\right)=$ $\Delta^{\operatorname{dim} A} H(A, N)(N=\sigma(A)-1)$; equality $\Leftrightarrow$ ann $\overline{A_{1}}=\overline{A_{N}}$. If equality holds, then $\bar{I}_{t} \neq 0(\alpha(\bar{I}) \leqslant t \leqslant N)$, whence, using $2(\mathrm{~b}, \mathrm{c}, \mathrm{d})$ :

$$
\begin{aligned}
\delta(A / I) & =\lambda(\bar{A} / \bar{I}) \leqslant \lambda(\bar{A})-(\sigma(\bar{A})-\alpha(\bar{I})) \\
& =\delta(A)-\sigma(A)+\alpha(I) \leqslant \delta(A)-1 .
\end{aligned}
$$

Remark. Observe that $\delta(A / I)=\delta(A)-1 \Rightarrow J \in \operatorname{Ass}(A)$ and $\delta(A / J)=1$, i.e., $\operatorname{Proj}(A / J)$ is a linear component of $\operatorname{Proj}(A)_{\text {red }}$. The existence of such a component is guaranteed if and only if $\operatorname{Proj}(A)$ is 0 -dimensional and has a $k$-rational point. That 
is, the natural domain of applicability of "Cayley-Bacharach" is that of 0-dimensional subschemes of $\mathbf{P}^{n}(k=\bar{k})$. Although such schemes may have "CayleyBacharach" without being arithmetically Gorenstein, we have

5. Theorem. Suppose that $A$ is reduced, $\operatorname{dim} A=1$, and every point of $\operatorname{Proj}(A)$ is $k$-rational. Then $A$ is Gorenstein if and only if the following two conditions are satisfied. (Let $N=\sigma(A)-1$.)

(a) (Symmetric Hilbert function)

$$
\Delta H(A, t)=\Delta H(A, N-t), \quad 0 \leqslant t \leqslant N .
$$

(b) (Cayley-Bacharach)

$$
\alpha(\operatorname{ann} \not h)=N \quad \text { for all } \not \in \operatorname{Ass}(A) .
$$

Proof. In view of 3 , we need only prove the sufficiency of (a) and (b). Let $C$ be the conductor of $A$ in its integral closure $B$. We shall prove that $A_{A_{1} A}$ is Gorenstein by verifying that $\lambda(B / C)=2 \lambda(A / C)[\mathbf{H K}, \mathrm{p}$. 32].

Identify $B$ as an $A$-algebra and an $\mathbf{N}$-graded $k$-algebra with $\oplus\{A / \not \mid \not \in \operatorname{Ass}(A)\}$. Note that $A / h \cong k[T]$ (graded $k$-algebra isomorphism). Under these circumstances $C$ is the ideal in $A$ (and in $B$ ), $\sum\{\operatorname{ann} \not h \mid \not \in \operatorname{Ass}(A)\}$ [O, Proposition 2.5]. So, by (b), $C_{t}=0$ for $0 \leqslant t<N$ and $\lambda\left(C_{N}\right) \geqslant \delta=\delta(A)=\operatorname{card}(\operatorname{Ass}(A))$. On the other hand, $\lambda\left(B_{t}\right)=\delta \quad(t \geqslant 0)$ and $\lambda\left(A_{N}\right)=H(A, N)=\delta$. Consequently, $A_{N}=B_{N}=C_{N}$, $\lambda(B / C)=N \delta$ and $\lambda(A / C)=\sum\{H(A, t) \mid 0 \leqslant t \leqslant N-1\}$. Now, $H(A, t)=$ $\sum\{\Delta H(A, j) \mid 0 \leqslant j \leqslant t\}=H(A, N)-\sum\{\Delta H(A, j) \mid t+1 \leqslant j \leqslant N\}$. Then, using (a),

$$
H(A, t)=\delta-\sum\{\Delta H(A, j) \mid 0 \leqslant j \leqslant N-1-t\}=\delta-H(A, N-1-t) .
$$

Consequently, $2 \lambda(A / C)=2\left(\sum\{H(A, t) \mid 0 \leqslant t \leqslant N-1\}\right)=N \delta=\lambda(B / C)$.

REMARKS. We do not know to what extent the hypothesis "reduced" can be eliminated from 5. In case $\lambda\left(A_{1}\right) \leqslant 3$, i.e., in case $\operatorname{Proj}(A)$ is a subscheme of $\mathbf{P}^{2}$, [DM] proves 5 without "reduced", and a stronger result than 5 with "reduced". That analysis depends heavily on the fact that, in $\mathbf{P}^{2}$,"arithmetically Gorenstein" = "complete intersection". 5 should also be compared with Stanley's characterization of Gorenstein domains among the CM domains [S, Theorem 4.4].

\section{REFERENCES}

[AG] Y. Aoyama and S. Goto, On the type of graded Cohen-Macaulay rings, J. Math. Kyoto Univ. 15 (1975), 19-23.

[DM] E. Davis and P. Maroscia, Complete intersections in $\mathbf{P}^{2}$, Proc. Conf. on Complete Intersections (Acireale, June 1983), Lecture Notes in Math., (in press).

[G] W. Gröbner, Über Irreduzibel Ideale in Kommutativen Ringen, Math. Ann. 110 (1934), 197-222.

[HK] J. Herzog and E. Kunz, Der Kanonische Modul eines Cohen-Macaulay-Rings, Lecture Notes in Math, Vol. 238, Springer-Verlag, Berlin, Heidelberg and New York, 1967.

[K] I. Kaplansky, Commutative rings, Allyn \& Bacon, Boston, Mass., 1970.

[N] M. Nagata, Local rings, Tracts in Pure and Appl. Math., No. 13, Interscience, New York and London, 1962. 
[O] F. Orecchia, Points in generic position and the conductor of curves with ordinary singularities, J. London Math. Soc. (2) 24 (1981), 85-96.

[PS] C. Peskine and L. Szpiro, Liaison des variétés algébriques. I, Invent. Math. 26 (1974), 271-302.

[SR] J. G. Semple and L. Roth, Introduction to algebraic geometry, Clarendon Press, Oxford, 1949.

[S] R. Stanley, Hilbert functions of graded algebras, Adv. in Math. 28 (1978), 57-83.

Department of Mathematics, SUNy, Center at Albany, Albany, New York 12222

DePartment of Mathematics, QueEn's University, Kingston K7L 3N6, Ontario, Canada

Department of Mathematics, Universita di Genova, Genova, Italy 